\title{
LA FAMILIA ANOREXÍGENA. UNA VISIÓN SISTÉMICA DE LOS TRASTORNOS DE LA CONDUCTA ALIMENTARIA
}

\section{RAQUEL DE ANTONIO PASTOR}

Enfermera especialista en salud mental. Unidad de Cuidados Psiquiátricos Prolongados. Instituto Psiquiátrico José Germain. Madrid.

\section{INTRODUCCIÓN}

Los trastornos de la conducta alimentaria (TCA) son un tipo de trastorno mental multifactorial cuyo mayor pico de incidencia se da en la adolescencia, y cuyo sexo más prevalente es el femenino ${ }^{1,2}$.

Además de los signos y síntomas más conocidos, como las restricciones alimentarias y las purgas, se han identificado ciertos factores familiares que ejercen una gran influencia en la aparición de estos trastornos como son las dificultades en la comunicación - especialmente, con la madre-, los antecedentes personales y familiares de obesidad y de dificultad en la alimentación previos al desarrollo de la enfermedad, así como la crianza en un medio sobreprotector $^{1-3}$.

Antes de la década de 1970, los TCA se consideraban enfermedades psicosomáticas y se excluía del tratamiento a la familia. Con el surgimiento de las terapias sistémicas, el abordaje terapéutico se modificó, ya que este nuevo enfoque trata de vincular el síntoma con el entorno, dando lugar a que la familia participe en el proceso de solución del conflicto,

Correspondencia: Raquel de Antonio Pastor Correo electrónico: raquelde.antonio@salud.madrid.org puesto que los síntomas son vistos como parte de las interacciones que se dan en el sistema familiar. Desde esta perspectiva, la familia no puede ser comprendida sin incluir su historia, mandatos, patrones de adaptación generacionales, creencias y expectativas, que, a su vez, influyen en la forma de percepción de una crisis y en la búsqueda de soluciones de esta ${ }^{1-4}$. Estas terapias sostienen que las dificultades familiares aparecen cuando una familia no ha podido solucionar y afrontar las tareas evolutivas normales y adaptarse a los nuevos requerimientos que el sistema exige $^{1,3}$.

Los autores sistémicos describen el síntoma como un resultado razonable de un fallo en la estructura familiar, explicado a través de términos como la confusión de roles y jerarquías, alianzas o coaliciones entre los subsistemas y un deterioro de las funciones ejecutivas; y proponen la existencia de ciertos patrones disfuncionales específicos que contribuyen al desarrollo y mantenimiento del trastorno. Algunas de las pautas descritas consisten en la fusión y rigidez en la familia, la dedicación al autosacrificio y la lealtad grupal frente a la diferenciación individual ${ }^{1,3,4}$. También afirman que no tener en cuenta la correspondencia entre el síntoma y el contexto familiar puede beneficiar la cronicidad de la enfermedad, 
donde el cuerpo sería la representación del descontento familiar. Por lo tanto, la familia es un factor importante del tratamiento del TCA, resultando fundamental incorporar en él al círculo familiar del individuo enfermo ${ }^{1-4}$.

\section{PRESENTACIÓN DEL CASO CLÍNICO}

Mujer de 14 años que acude a la unidad de adolescentes derivada de su centro de salud mental (CSM) de referencia para el ingreso por clínica de TCA, con pérdida de más de medio kilo en la última semana; un total de $15 \mathrm{~kg}$ en los cuatro meses previos (índice de masa corporal [IMC] de $15,4 \mathrm{~kg} / \mathrm{m}^{2}$ en el momento de la valoración).

La familia refiere que comenzó con esta clínica en agosto de 2017. Comía acompañada, pero empezó a dejar de comer progresivamente y sin ocultarlo ni esconder comida. Al principio, no dieron importancia a la restricción alimentaria. Niegan estresores psicobiográficos que hayan podido precipitar la clínica. Sin alergias conocidas ni antecedentes de interés. Ausencia de antecedentes psiquiátricos previos. Niega el consumo de tóxicos.

A la valoración, se muestra consciente, orientada en las tres esferas y colaboradora. Resonancia conservada. Delgadez extrema y palidez cutánea. Ánimo eutímico sin clínica de trastorno afectivo mayor. Niega ansiedad, aunque impresiona de angustia contenida. Discurso parco y escueto, pero coherente. No se aprecian alteraciones sensoperceptivas ni formales en el curso ni en el contenido del pensamiento. No se observa clínica delirante ni disociativa. Niega ideación autolítica. Ausencia de autolesiones. Dismorfofobia abdominal. Conducta alimentaria restrictiva sin purgas asociadas, con pérdida ponderal importante (IMC: 15,4 kg/m²). Leve insomnio de conciliación. Juicio de la realidad y capacidad volitiva conservados.

- Información familiar: hermana melliza con antecedentes de hipocondría y ansiedad, ya de alta por parte de su CSM de referencia, y en normopeso. Padres no fumadores. Ambos trabajan por la mañana, pero acuden a actividades lúdicas por las tardes, por separado, regresando a las 22:00 h al domicilio familiar.

- Historia académica: buen rendimiento académico; no ha repetido curso. Actualmente, cursa $3 .^{\circ}$ de la Enseñanza Secundaria Obligatoria. Niega problemas en el colegio o a nivel social.

- Ambiente social: pertenece al grupo de amistades de su hermana.

- Intereses personales: le gusta tocar la guitarra y jugar al voleibol. Antes estaba en un equipo; ahora solo juega de vez en cuando en el instituto. Cuando se le pregunta por lo que le gustaría hacer en el futuro, refiere que no se lo ha planteado aún.

Expresa que sentía temor por volver a verse como en $6 .^{\circ}$ de Primaria (sobrepeso). Paulatinamente, fue restringiendo alimentos calóricos hasta restringir la ingesta de líquidos ( «poco a poco, empecé a dejar de tener hambre y, más tarde, incluso dejé de tener sed»). Sus padres no le dieron importancia hasta que vieron una notable pérdida ponderal pasado un mes. Comen juntos en familia.

Se explora con ella la relación entre sus padres. «Antes era mejor. Ahora discuten mucho. Mi hermana y yo discutimos mucho y ellos acaban metiéndose y discutiendo por nosotras. Eso me hace sentir culpable. Temo que se divorcien».

Se le pregunta por el inicio del problema con la comida. Al principio, no sabe datarlo; posteriormente, al preguntarle si percibe si el desgaste de la relación de sus padres fue antes o después del inicio de su problema con la comida, responde: «fue algo antes; cada vez discutían más por tonterías y por mi hermana y por mí, y yo me sentía culpable. Pero desde que estoy aquí, vienen los dos a verme y parece que se llevan mejor; se muestran más cercanos el uno con el otro y más cariñosos. Yo los veo mejor». 


\section{VALORACIÓN DE ENFERMERÍA}

La valoración de enfermería se lleva a cabo siguiendo los patrones funcionales de Marjory Gordon y utilizando como herramientas la observación en la unidad y la entrevista semiestructurada tanto a la paciente como a la familia (tabla 1 ).

\section{DIAGNÓSTICOS DE ENFERMERÍA Y PLANIFICACIÓN}

Los diagnósticos de enfermería se formularon de acuerdo con la NANDA (North American Nursing Diagnosis Association) ${ }^{5}$, y la clasificación de resultados e intervenciones y actividades de enfermería se basaron, respectivamente, en la NOC (Nursing Outcomes Classification) ${ }^{6}$ y la NIC (Nursing Interventions (lassification) ${ }^{7}$ (tabla 2).

\section{EVALUACIÓN DEL PLAN DE CUIDADOS Y RESULTADOS}

Desde el principio, la paciente se compromete a completar todas las ingestas para mantener privilegios de visitas y ampliarlos a salidas. En la primera semana, se muestra aislada, pero cumplidora. Estuvo poco comunicativa durante las entrevistas, con respuestas monosilábicas y discurso inducido, y refiriendo insomnio de conciliación y mantenimiento, así como fatiga y dificultad para la concentración.

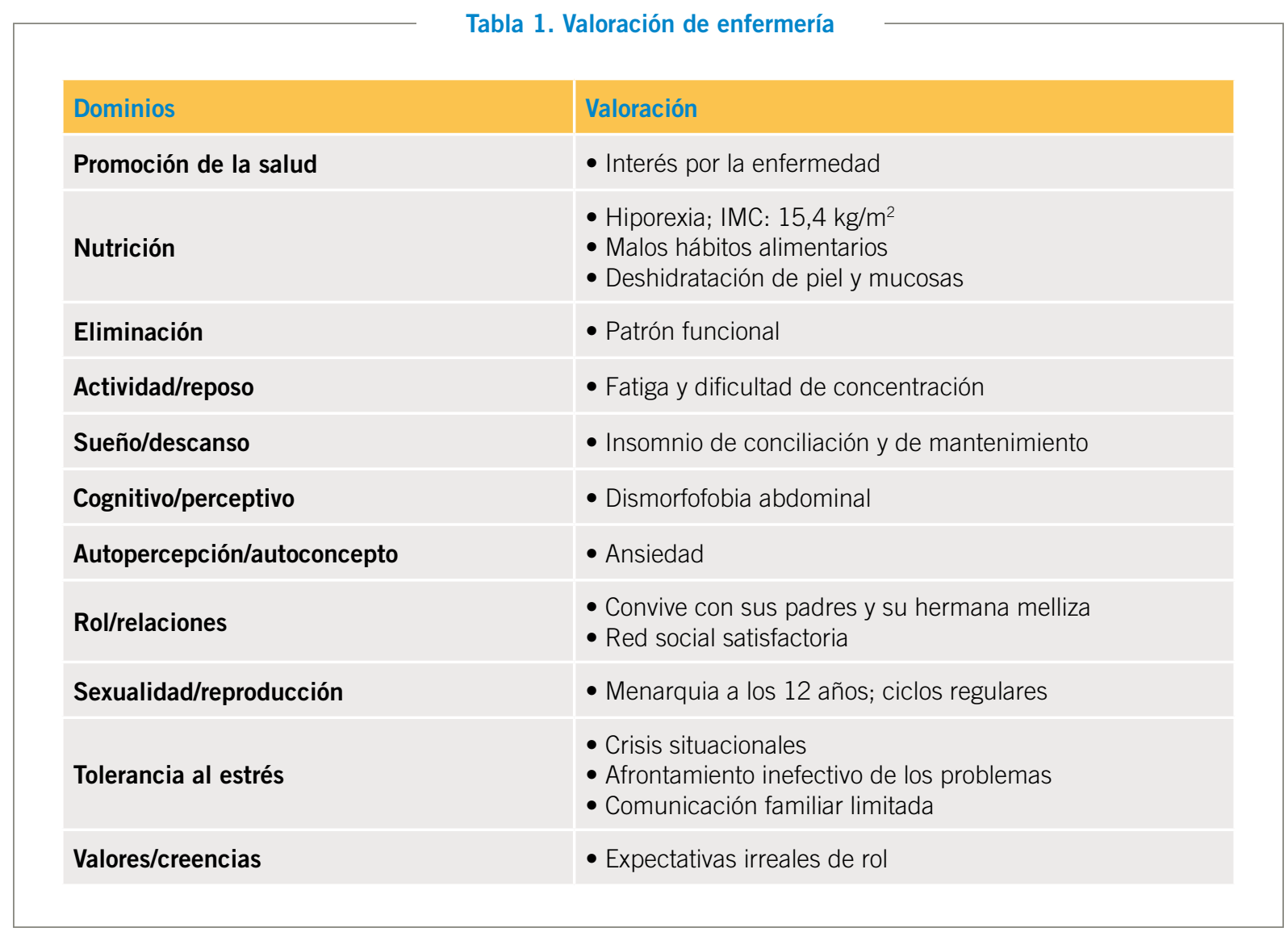

IMC: índice de masa corporal. 
00055 Desempeño ineficaz del rol r/c expectativas irreales del rol.

1305 Adaptación psicosocial: cambio de vida

- 130507 Expresiones de sentimientos permitidos.

- 130501 Establecimiento de objetivos realistas.

NOC

\section{Superación de problemas}

- 130203 Verbaliza sensación de control.

- 130205 Verbaliza aceptación de la situación.

- 130208 Se adapta a los cambios en desarrollo.

PI: 2; PD: 4; PA: 3

PI: 1; PD: 4; PA: 3

PI: 1; PD: 5; PA: 4

- 130212 Utiliza estrategias de superación efectivas.

- 130215 Busca ayuda profesional de forma apropiada.

\section{Acuerdo con el paciente}

- Ayudar al paciente a determinar objetivos realistas, que puedan conseguirse.

- Establecer objetivos en términos positivos.

- Ayudar al paciente a desarrollar un plan para cumplir con los objetivos.

\section{Potenciación de roles}

- Ayudar al paciente a identificar los diversos papeles en la vida.

- Ayudar al paciente a identificar los roles habituales en la familia.

- Ayudar al paciente a identificar períodos de transición de papeles a lo largo de la vida.

- Ayudarle a identificar las conductas necesarias para el cambio de roles a roles nuevos.

NIC

- Ayudar a los niños mayores a aceptar la dependencia de los padres mayores y los cambios de rol implicados, si procede.

- Facilitar la discusión sobre la adaptación de los roles de la familia para compensar los cambios de rol del miembro enfermo.

\section{Intervención en caso de crisis}

- Disponer un ambiente de apoyo.

- Determinar si el paciente presenta riesgo de seguridad para sí mismo o para otros.

- Tener las precauciones necesarias para salvaguardar al paciente u otras personas con riesgo de lesiones físicas.

- Favorecer la expresión de sentimientos de una forma no destructiva.

00118 Trastorno de la imagen corporal r/c enfermedad psiquiátrica m/p percepción distorsionada de la realidad.

\section{Identidad}

- 120201 Verbaliza afirmaciones de identidad personal.

NOC $\quad 120207$ Realiza roles sociales.

- 120208 Verbaliza un sistema de valores propio.

PI: 1; PD: 4; PA: 4

- 120210 Cuestiona imágenes negativas sobre sí mismo.

PI: 2; PD: 4; PA: 3

PI: 2; PD: 4; PA: 4

PI: 2; PD: 4; PA: 3

\section{Potenciación de la conciencia de sí mismo}

- Animar al paciente a reconocer y discutir sus pensamientos y sentimientos.

- Ayudar al paciente a identificar los valores que contribuyen al concepto de sí mismo.

NIC

- Ayudar al paciente a identificar los sentimientos habituales que tiene de sí mismo.

- Facilitar la identificación de sus formas de respuesta habitual a diversas situaciones por parte del paciente.

- Ayudar al paciente a identificar el impacto que tiene la enfermedad sobre el concepto de sí mismo.

- Ayudar al paciente a identificar los sentimientos de culpa. 
Tabla 2. Diagnósticos de enfermería y planificación (cont.)

00162 Disposición para mejorar la gestión de la propia salud m/p elecciones de la vida diaria que son apropiadas para satisfacer los objetivos de prevención o tratamiento.

1847 Conocimientos: manejo de la enfermedad crónica

NOC

- 184719 Importancia de cumplir el régimen terapéutico.

- 160914 Equilibrio entre tratamiento, ejercicio, trabajo, ocio y nutrición.

- 160915 Busca consejo de un profesional sanitario cuando es necesario.
PI: 3; PD: 5; PA: 3

PI: 3; PD: 5; PA: 3

PI: 3; PD: 5; PA: 4

\section{(6610) Identificación de riesgos}

- Determinar la presencia y calidad del apoyo familiar.

- Determinar el nivel de funcionamiento pasado y actual.

- Determinar los recursos comunitarios adecuados.

NIC

- Proceder a derivar a otro personal/institución.

(5602) Enseñanza: proceso de enfermedad

- Describir el proceso de enfermedad.

- Describir signos y síntomas comunes de la enfermedad.

- Describir el fundamento de las recomendaciones de control/terapia/tratamiento.

- Instruir al paciente y cuidador sobre cuáles son los signos y síntomas de los que debe informar.

m/p: manifestado por; NIC: intervenciones enfermeras; NOC: criterios de resultados de enfermería; PA: puntuación alcanzada; PD: puntuación diana; PI: puntuación al ingreso; r/c: relacionado con.

Durante la segunda semana, se mostró más comunicativa que en la previa, aunque manteniendo un discurso parco y mayoritariamente inducido. Continuó cumplidora en cuanto a ingestas y toma de medicación y verbalizó sentirse con más energía y mejor ánimo. Solicitó por sí misma que se autorizara a su hermana a acudir a las visitas, permitiéndole salidas para que pudiera verla, dado que, por ser menor de edad, no estaba permitido su acceso a la unidad.

En la tercera semana, se mostró más sonriente y espontánea, refiriendo mejora en el estado de ánimo, en la concentración y en el sueño. Mostraba preocupación por la enfermedad ( ¿¿Cuándo voy a dejar de verme mal?») y trataba de buscar soluciones («¿Cómo sé que, si me veo mal, es por la enfermedad?»). Se le dijo en diferentes ocasiones que, conforme fuera ganando peso, la distorsión de la imagen corporal iría mejorando. No obstante, se le indicó que mantuviera un IMC mínimo y tomara un patrón externo (por ejemplo, su hermana, a la cual veía bien y mantenía normopeso).
Finalmente, en la cuarta semana, se le ofreció un tratamiento ambulatorio intensivo al alta en un hospital de día para trabajar los aspectos más psicoterapéuticos de la enfermedad, a lo cual accedió. Esta propuesta también se le comunicó a la madre en una entrevista, quien también se mostró conforme, momento en el que se comenzó la coordinación con el dispositivo indicado y se procedió al alta.

\section{BIBLIOGRAFÍA}

1. Losada AV, Whittingslow M. Técnicas de la terapia sistémica en trastornos de la conducta alimentaria. Revista Borromeo. 2013;(4):393-424.

2. Cerniglia L, Cimino S, Tafà M, Marzilli E, Ballarotto G, Bracaglia $F$. Family profiles in eating disorders: family functioning and psychopathology. Psychol Res Behav Manag. 2017;10:305-12.

3. Wallis A, Rhodes P, Dawson L, Miskovic-Wheatley J, Madden S, Touyz S. Relational containment: exploring the effect of family-based treatment for anorexia on familial relationships. J Eat Disord. 2017;5:27.

4. Wallis A, Miskovic-Wheatley J, Madden S, Rhodes P, Crosby RD, Cao L, et al. How does family functioning 
effect the outcome of family based treatment for adolescents with severe anorexia nervosa? J Eat Disord. 2017; 5:55.

5. Herdman T, Kamitsuru S. NANDA International, Inc. Diagnósticos enfermeros. 11. ${ }^{a}$ ed. Barcelona: Elsevier España; 2019.
6. Moorhead S, Swanson E, Johnson M, Maas M. Clasificación de resultados de enfermería (NOC). 6. ${ }^{a}$ ed. Barcelona: Elsevier España; 2018.

7. Butcher H, Bulechek G, Dochterman J, Wagner C. Clasificación de intervenciones de enfermería (NIC). 7. ${ }^{a}$ ed. Barcelona: Elsevier; 2018. 